\title{
Asymptotic behavior of the Riemannian Heisenberg group and its horoboundary
}

\author{
Enrico Le Donne $^{1}$ (D) . Sebastiano Nicolussi-Golo ${ }^{1}$ (D) \\ Andrea Sambusetti ${ }^{2}$
}

Received: 19 March 2016 / Accepted: 4 October 2016 / Published online: 15 October 2016

(C) Fondazione Annali di Matematica Pura ed Applicata and Springer-Verlag Berlin Heidelberg 2016

\begin{abstract}
The paper is devoted to the large-scale geometry of the Heisenberg group $\mathbb{H}$ equipped with left-invariant Riemannian metrics. We prove that two such metrics have bounded difference if and only if they are asymptotic, i.e., their ratio goes to one at infinity. Moreover, we show that for every left-invariant Riemannian metric $d$ on $\mathbb{H}$ there is a unique subRiemannian metric $d^{\prime}$ for which $d-d^{\prime}$ goes to zero at infinity, and we estimate the rate of convergence. As a first immediate consequence, we get that the Riemannian Heisenberg group is at bounded distance from its asymptotic cone. The second consequence, which was our aim, is the explicit description of the horoboundary of the Riemannian Heisenberg group.
\end{abstract}

Keywords Heisenberg group · Horoboundary · Asymptotic cone · Riemannian geometry · SubRiemannian geometry

Mathematics Subject Classification $20 \mathrm{~F} 69 \cdot 53 \mathrm{C} 23 \cdot 53 \mathrm{C} 17$

\section{Contents}

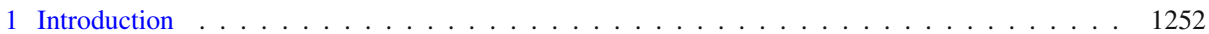

1.1 Detailed results . . . . . . . . . . . . . . . . . . . . . . . 1253

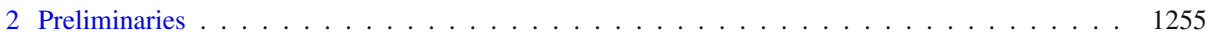

2.1 Definitions . . . . . . . . . . . . . . . . . . . . . . . . . . . 1255

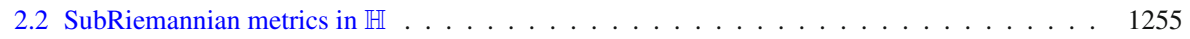

2.3 Balayage area and lifting of curves . . . . . . . . . . . . . . . . . . . . . . . 1257

E.L.D. has been supported by the Academy of Finland Project no. 288501. S.N.G. has been supported by the People Programme (Marie Curie Actions) of the European Union's Seventh Framework Programme FP7/2007-2013/ under REA Grant agreement no. 607643.

Sebastiano Nicolussi-Golo sebastiano2.72@fastmail.fm

1 University of Jyvaskyla, Jyväskylä, Finland

2 Sapienza Università di Roma, Rome, Italy 
3 Comparison between strictly subRiemannian metrics . . . . . . . . . . . . . . . . . . . . . . 1258

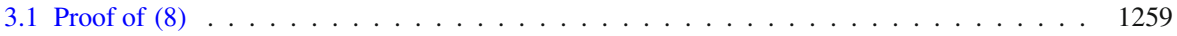

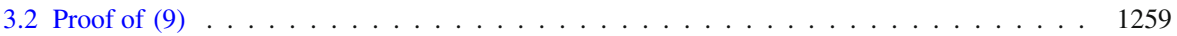

4 Comparison between Riemannian and strictly subRiemannian metrics . . . . . . . . . . . . . . 1261

5 The horoboundary . . . . . . . . . . . . . . . . . . . . . . . . . 1265

5.1 Concluding remarks . . . . . . . . . . . . . . . . . . . . . . . . . . . 1268

Appendix: Length-minimizing curves for $d_{C C}$ and $d_{R} \ldots \ldots \ldots \ldots$. . . . . . . . . . . . . . . 1269

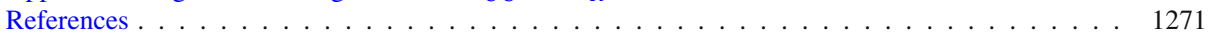

\section{Introduction}

In large-scale geometry, various notions of space at infinity have received special interest for differently capturing the asymptotic geometric behavior. Two main examples of spaces at infinity are the asymptotic cone and the horoboundary. The description of the asymptotic cone for finitely generated groups is a crucial step in the algebraic characterization of groups of polynomial growth $[4,12,21,23,30,33]$. The notion of horoboundary has been formulated by Gromov [11], inspired by the seminal work of Busemann on the theory of parallels on geodesic spaces [6]. The horoboundary has a fully satisfying visual description in the framework of $C A T(0)$-spaces and of Gromov-hyperbolic spaces [2,3,13]. It plays a major role in the study of dynamics and rigidity of negatively curved spaces [13,15, 22, 24, 27,29]. The visual-boundary description breaks down for non-simply connected manifolds [9] and when the curvature has variable sign, as we will make evident for the Riemannian Heisenberg group.

This paper contributes to the study of the asymptotic geometry of the simplest nonAbelian nilpotent group: the Heisenberg group. The asymptotic cone of the Heisenberg group equipped with a left-invariant Riemannian metric $d_{R}$ is the Heisenberg group equipped with a Carnot-Carathéodory metric $d_{C C}$, see [23] and also [4]. Our contribution is a finer analysis of the asymptotic comparison of $d_{R}$ and $d_{C C}$. This leads to the explicit knowledge of the (Riemannian) horoboundary. We remark that the Heisenberg group is not hyperbolic; hence, one does not consider its visual boundary.

We recall the definition of horoboundary. Let $(X, d)$ be a metric space. We consider the space of continuous real functions $\mathscr{C}(X)$ endowed with the topology of uniform convergence on compact sets. We denote by $\mathscr{C}(X) / \mathbb{R}$ the quotient with respect to the subspace of constant functions. The map $x \mapsto d(x, \cdot)$ induces an embedding $X \hookrightarrow \mathscr{C}(X) / \mathbb{R}$. The horoboundary of $X$ is defined as $\partial_{h} X:=\bar{X} \backslash X \subset \mathscr{C}(X) / \mathbb{R}$. See Sect. 5 for a detailed exposition.

The horoboundary has been investigated for finite-dimensional normed vector spaces [31], for Hilbert geometries [32], and for infinite graphs [34]. For non-simply connected, negatively curved manifolds, it has been studied in [9]. Nicas and Klein computed the horoboundary of the Heisenberg group when endowed with the Korany metric in [17] and with the metric $d_{C C}$ in [18].

We will show that the horoboundary of the Heisenberg group endowed with a left-invariant Riemannian metric $d_{R}$ coincides with the second case studied by Nicas and Klein, see Corollary 1.4. This will be an immediate consequence of our main result Theorem 1.3, which implies that the difference $d_{R}-d_{C C}$ converges to zero when evaluated on points $\left(p, q_{n}\right)$ with $q_{n}$ being a sequence that leaves every compact set.

Remark 1.1 Another term for Carnot-Carathéodory metric is subRiemannian metric. In this paper, we should discuss subRiemannian metrics that may actually be Riemannian. Therefore, we follow the convention that subRiemannian geometry includes as a particular case 
Riemannian geometry. This is in agreement with several established references in the field, see $[1,16,26]$. In the presence of a subRiemannian metric that is not Riemannian, we shall use the term strictly subRiemannian.

\subsection{Detailed results}

The Heisenberg group $\mathbb{H}$ is the simply connected Lie group whose Lie algebra $\mathfrak{h}$ is generated by three vectors $X, Y, Z$ with only nonzero relation $[X, Y]=Z$. A left-invariant Riemannian metric $d$ on $\mathbb{H}$ is determined by a scalar product $g$ on $\mathfrak{h}$; a left-invariant strictly subRiemannian metric $d$ is induced by a bracket generating plane $V \subset \mathfrak{h}$ and a scalar product $g$ on $V$ (see Sect. 2 for detailed exposition). In both cases, we say that $d$ is subRiemannian with horizontal space $(V, g)$, where $\operatorname{dim} V$ is either 2 or 3 .

We are interested in the asymptotic comparison between these metrics. Given two leftinvariant subRiemannian metrics $d$ and $d^{\prime}$ on $\mathbb{H}$, we deal with three asymptotic behaviors, in ascending order of strength, each of which defines an equivalence relation among subRiemannian metrics:

(i) $\lim _{d(p, q) \rightarrow \infty} \frac{d(p, q)}{d^{\prime}(p, q)}=1$;

(ii) There exists $c>0$ such that $\left|d(p, q)-d^{\prime}(p, q)\right|<c$, for all $p, q$;

(iii) $\lim _{d(p, q) \rightarrow \infty}\left|d(p, q)-d^{\prime}(p, q)\right|=0$.

A first example of the implication $(i) \Rightarrow$ (ii) was proved by Burago in [5] for $\mathbb{Z}^{n}$-invariant metrics $d$ on $\mathbb{R}^{n}$, by showing that $d$ and the associated stable norm stay at bounded distance from each other. This result has been extended quantitatively for $\mathbb{Z}^{n}$-invariant metrics on geodesic metric spaces in [8]. Gromov and Burago asked for other interesting cases where the same implication holds. Another well-known case where (i) is equivalent to (ii) is that of hyperbolic groups. Beyond Abelian and hyperbolic groups, Krat proved the equivalence for word metrics on the discrete Heisenberg group $\mathbb{H}(\mathbb{Z})[19]$. For general subFinsler metrics on Carnot groups, it has been proven in [4], following [28], that (i) is equivalent to the fact that the projections onto $\mathbb{H} /[\mathbb{H}, \mathbb{H}]$ of the corresponding unit balls coincide, see (c) below. Our first result shows that this last condition is equivalent to each one of (i) and (ii) in the case of the Heisenberg group endowed with subRiemannian metrics.

Theorem 1.2 Let $d$ and $d^{\prime}$ be two left-invariant subRiemannian metrics on $\mathbb{H}$ whose horizontal spaces are $(V, g)$ and $\left(V^{\prime}, g^{\prime}\right)$, respectively. Let $\pi: \mathfrak{h} \rightarrow \mathfrak{h} /[\mathfrak{h}, \mathfrak{h}]$ be the quotient projection and $\hat{\pi}: \mathbb{H} \rightarrow \mathfrak{h} /[\mathfrak{h}, \mathfrak{h}]$ the corresponding group morphism. Then, the following assertions are equivalent:

(a) there exists $c>0$ such that $\left|d(p, q)-d^{\prime}(p, q)\right|<c$, for all $p, q$;

(b) $\frac{d(p, q)}{d^{\prime}(p, q)} \rightarrow 1$ when $d(p, q) \rightarrow \infty$;

(c) $\hat{\pi}(\{p \in \mathbb{H}: d(0, p) \leq R\})=\hat{\pi}\left(\left\{p \in \mathbb{H}: d^{\prime}(0, p) \leq R\right\}\right)$, for all $R>0$, here 0 denotes the neutral element of $\mathbb{H}$;

(d) $\pi(\{v \in V: g(v, v) \leq 1\})=\pi\left(\left\{v^{\prime} \in V^{\prime}: g^{\prime}\left(v^{\prime}, v^{\prime}\right) \leq 1\right\}\right)$;

(e) there exists a scalar product $\bar{g}$ on $\mathfrak{h} /[\mathfrak{h}, \mathfrak{h}]$ such that both

$$
\left.\pi\right|_{V}:(V, g) \rightarrow(\mathfrak{h} /[\mathfrak{h}, \mathfrak{h}], \bar{g}) \text { and }\left.\pi\right|_{V^{\prime}}:\left(V^{\prime}, g^{\prime}\right) \rightarrow(\mathfrak{h} /[\mathfrak{h}, \mathfrak{h}], \bar{g})
$$

are submetries, i.e., they map balls to balls.

Next, we prove that in every class of the equivalence relation (iii) there is exactly one strictly subRiemannian metric. To every left-invariant subRiemannian metric $d$, we define the associated asymptotic metric $d^{\prime}$ as follows. If $d$ is Riemannian defined by a scalar product 
$g$ on $\mathfrak{h}$, then $d^{\prime}$ is the strictly subRiemannian metric for which the horizontal space $V$ is $g$ orthogonal to $[\mathfrak{h}, \mathfrak{h}]$ and the scalar product is $\left.g\right|_{V}$. If $d$ is strictly subRiemannian, then $d^{\prime}=d$.

Theorem 1.3 Let $d$ and $d^{\prime}$ be two left-invariant subRiemannian metrics on $\mathbb{H}$. Their associated asymptotic metrics are the same if and only if

$$
\lim _{d(p, q) \rightarrow \infty}\left|d(p, q)-d^{\prime}(p, q)\right|=0 .
$$

Moreover, if (1) holds, then there is $C>0$ such that

$$
\left|d(p, q)-d^{\prime}(p, q)\right| \leq \frac{C}{d(p, q)}, \quad \forall p, q \in \mathbb{H} .
$$

We remark that the estimate (2) in Theorem 1.3 is sharp, as we will show in Remark 4.2.

The above result can be interpreted in terms of asymptotic cones. Namely, if $d$ is a leftinvariant Riemannian metric on $\mathbb{H}$ and $d^{\prime}$ is the associated asymptotic metric, then $\left(\mathbb{H}, d^{\prime}\right)$ is the asymptotic cone of $(\mathbb{H}, d)$. For the analogous result in arbitrary nilpotent groups, see [23]. By Theorem 1.3, more is true: $(\mathbb{H}, d)$ is at bounded distance from $\left(\mathbb{H}, d^{\prime}\right)$. Notice that this consequence cannot be deduced by the similar results for discrete subgroups of the Heisenberg group in [19] and [10], because the word metric is only quasi-isometric to the Riemannian one. Moreover, we remark that there are examples of nilpotent groups of step two that are not at bounded distance from their asymptotic cone, see [4].

We now focus on the horoboundary. As a consequence of Theorem 1.3 and of the results of Klein-Nikas [18], we get:

Corollary 1.4 If $d_{R}$ is a left-invariant Riemannian metric on $\mathbb{H}$ with associated asymptotic metric $d_{C C}$, then the horoboundary of $\left(\mathbb{H}, d_{R}\right)$ coincides with the horoboundary of $\left(\mathbb{H}, d_{C C}\right)$; hence, it is homeomorphic to a two-dimensional closed disk $\bar{D}^{2}$.

More precisely, let $g$ be the scalar product of $d_{R}$ on $\mathfrak{h}$ and $W \subset \mathfrak{h}$ the orthogonal plane to $[\mathfrak{h}, \mathfrak{h}]$. Define the norm $\|w\|:=\sqrt{g(w, w)}$ on $W$. Fix a orthonormal basis $(X, Y)$ for $W$ and set $Z:=[X, Y] \in[\mathfrak{h}, \mathfrak{h}]$, so that $(X, Y, Z)$ is a basis of $\mathfrak{h}$. We identify $\mathfrak{h} \simeq \mathbb{H}$ via the exponential map, which is a global diffeomorphism. So, we write $p=w+z Z$ with $w \in W$ and $z \in \mathbb{R}$ for any point $p \in \mathbb{H}$. We say that a sequence of points $\left\{p_{n}\right\}_{n \in \mathbb{N}} \subset \mathbb{H}$ diverges if it leaves every compact set. Moreover, we shall use the following terminology for a diverging sequence of the form $p_{n}=w_{n}+z_{n} Z$ :

(1) vertical divergence, if there exists $M<\infty$ such that $\left\|w_{n}\right\|<M$ for all $n$;

(2) non-vertical divergence with quadratic rate $v \in[-\infty,+\infty]$, if $w_{n}$ diverges and ${ }^{1}$ $\lim _{n \rightarrow \infty} \frac{z_{n}}{4\left\|w_{n}\right\|^{2}}=-v$.

Then, according to [18] (see Corollary 5.6, 5.9 and 5.13 therein), we deduce the following description of the Riemannian horofunctions:

(v): a vertically diverging sequence $p_{n}=w_{n}+z_{n} Z$ converges to a horofunction $h$ if and only if $w_{n} \rightarrow w_{\infty}$, and in this case

$$
h(w+z Z)=\left\|w_{\infty}\right\|-\left\|w_{\infty}-w\right\| ;
$$

(nv): a non-vertically diverging sequence $p_{n}=w_{n}+z_{n} Z$ with quadratic rate $v$ converges to a horofunction $h$ if and only if $\frac{w_{n}}{\left\|w_{n}\right\|} \rightarrow \hat{w}$, and then

$$
h(w+z Z)=g\left(R_{\vartheta}(-\hat{w}), w\right)
$$

1 From the paper [18], there is an extra 4 and a change of sign due to our different choices of coordinates. 
where $R_{\vartheta}$ is the anticlockwise rotation in $W$ of angle $\vartheta=\mu^{-1}(\nu)$, and $\mu:[-\pi, \pi] \rightarrow \overline{\mathbb{R}}$ is the extended Gaveau function

$$
\mu(\vartheta):=\frac{\vartheta-\sin \vartheta \cos \vartheta}{\sin ^{2}(\vartheta)} .
$$

Moreover, all the horofunctions of $\left(\mathbb{H}, d_{R}\right)$ are of type (v) or (nv), by Theorem 5.16 in [18]; it is also clear that neither is of both types.

In Sect. 5, we will also determine the Busemann points of $\partial_{h}\left(\mathbb{H}, d_{R}\right)$, that is those horofunctions obtained by points diverging along quasi-geodesics (see Definition 5.1). We obtain, as in the subRiemannian case:

Corollary 1.5 The Busemann points of $\left(\mathbb{H}, d_{R}\right)$ are the horofunctions of type (nv) and can be identified to the boundary of the disk $\bar{D}^{2}$.

The paper is organized as follows. In Sect. 2, we introduce the main objects and their basic properties. In Sect. 3, we estimate the difference between any two strictly subRiemannian left-invariant metrics on $\mathbb{H}$. In Sect. 4, we compare any Riemannian left-invariant metric on $\mathbb{H}$ and its associated asymptotic metric. At the end of the section, we shall prove Theorems 1.2 and 1.3. In Sect. 5, we concentrate on the horofunctions and we prove Corollaries 1.4 and 1.5. "Appendix" is devoted to the explicit description of subRiemannian geodesics.

\section{Preliminaries}

\subsection{Definitions}

The first Heisenberg group $\mathbb{H}$ is the connected, simply connected Lie group associated with the Heisenberg Lie algebra $\mathfrak{h}$. The Heisenberg Lie algebra $\mathfrak{h}$ is the only three- dimensional nilpotent Lie algebra that is not commutative. It can be proven that, for any two linearly independent vectors $X, Y \in \mathfrak{h} \backslash[\mathfrak{h}, \mathfrak{h}]$, the triple $(X, Y,[X, Y])$ is a basis of $\mathfrak{h}$ and $[X,[X, Y]]=$ $[Y,[X, Y]]=0$.

We denote by $\omega_{\mathbb{H}}: T \mathbb{H} \rightarrow \mathfrak{h}$ the left-invariant Maurer-Cartan form. Namely, denoting by 0 the neutral element of $\mathbb{H}$ and identifying $\mathfrak{h}$ with $T_{0} \mathbb{H}$, we have $\omega_{\mathbb{H}}(v):=\mathrm{d} L_{p}^{-1} v$ for $v \in T_{p} \mathbb{H}$, where $L_{p}$ is the left translation by $p$.

Let $\pi: \mathfrak{h} \rightarrow \mathfrak{h} /[\mathfrak{h}, \mathfrak{h}]$ be the quotient projection. Notice that $\mathfrak{h} /[\mathfrak{h}, \mathfrak{h}]$ is a commutative two-dimensional Lie algebra. So the map $\pi$ induces a Lie group epimorphism $\hat{\pi}: \mathbb{H} \rightarrow$ $\mathfrak{h} /[\mathfrak{h}, \mathfrak{h}] \simeq \mathbb{H} /[\mathbb{H}, \mathbb{H}]$.

\subsection{SubRiemannian metrics in $\mathbb{H}$}

Let $V \subset \mathfrak{h}$ be a bracket generating subspace. We have only two cases: either $V=\mathfrak{h}$ or $V$ is a plane and $\mathfrak{h}=V \oplus[\mathfrak{h}, \mathfrak{h}]$. In both cases, the restriction of the projection $\left.\pi\right|_{V}: V \rightarrow \mathfrak{h} /[\mathfrak{h}, \mathfrak{h}]$ is surjective. Let $g$ be a scalar product on $V$ and set the corresponding norm $\|v\|:=\sqrt{g(v, v)}$ for $v \in V$.

An absolutely continuous curve $\gamma:[0,1] \rightarrow \mathbb{H}$ is said horizontal if $\omega_{\mathbb{H}}\left(\gamma^{\prime}(t)\right) \in V$ for almost every $t$. For a horizontal curve, we have the length

$$
\ell(\gamma):=\int_{0}^{1}\left\|\omega_{\mathbb{H}}\left(\gamma^{\prime}(t)\right)\right\| \mathrm{d} t .
$$


A subRiemannian metric $d$ is hence defined as

$$
d(p, q):=\inf \{\ell(\gamma): \gamma \text { horizontal curve from } p \text { to } q\} .
$$

SubRiemannian metrics on $\mathbb{H}$ are complete, geodesic, and left-invariant. They are either Riemannian, when $V=\mathfrak{h}$, or strictly subRiemannian, when $\operatorname{dim} V=2$. The pair $(V, g)$ is called the horizontal space of $d$.

Since $\left.\pi\right|_{V}: V \rightarrow \mathfrak{h} /[\mathfrak{h}, \mathfrak{h}]$ is surjective, it induces a norm $\|\cdot\|$ on $\mathfrak{h} /[\mathfrak{h}, \mathfrak{h}]$ such that $\pi:(V,\|\cdot\|) \rightarrow(\mathfrak{h} /[\mathfrak{h}, \mathfrak{h}],\|\cdot\|)$ is an submetry, i.e., for all $w \in \mathfrak{h} /[\mathfrak{h}, \mathfrak{h}]$ it holds $\|w\|=$ $\inf \{\|v\|: \pi(v)=w\}$. Here we use the same notation for norms on $V$ and on $\mathfrak{h} /[\mathfrak{h}, \mathfrak{h}]$, because there will be no possibility of confusion. The norm on $\mathfrak{h} /[\mathfrak{h}, \mathfrak{h}]$ is characterized by

$$
\pi(\{v \in V:\|v\| \leq R\})=\{w \in \mathfrak{h} /[\mathfrak{h}, \mathfrak{h}]:\|w\| \leq R\},
$$

for all $R>0$.

Proposition 2.1 Let $d$ be subRiemannian metric on $\mathbb{H}$ with horizontal space $(V, g)$. Then, for all $R>0$

$$
\pi(\{v \in V:\|v\| \leq R\})=\hat{\pi}(\{p \in \mathbb{H}: d(0, p) \leq R\}) .
$$

In particular, $\hat{\pi}:(\mathbb{H}, d) \rightarrow(\mathfrak{h} /[\mathfrak{h}, \mathfrak{h}],\|\cdot-\cdot\|)$ is a submetry, i.e., for all $v, w \in \mathfrak{h} /[\mathfrak{h}, \mathfrak{h}]$

$$
\|v-w\|=\inf \{d(p, q): \hat{\pi}(p)=v, \hat{\pi}(q)=w\} .
$$

Proof $€$ Let $v \in V$ with $\|v\| \leq R$. Set $\gamma(t):=\exp (t v)$. Then, $\gamma:[0,1] \rightarrow \mathbb{H}$ is a horizontal curve with $d(0, \exp (v)) \leq \ell(\gamma)=\|v\| \leq R$. Since $\hat{\pi}(\exp (v))=\pi(v)$, then we have proven this inclusion.

$\bigcap$ Let $p \in \mathbb{H}$ with $d(0, p) \leq R$ and let $\gamma:[0, T] \rightarrow \mathbb{H}$ be a $d$-length-minimizing curve from 0 to $p$ parametrized by arc length, so $T=d(0, p)$. Then, $\hat{\pi} \circ \gamma:[0, T] \rightarrow \mathfrak{h} /[\mathfrak{h}, \mathfrak{h}]$ is a curve from 0 to $\pi(p)$ and

$$
\begin{aligned}
\|\hat{\pi}(p)\| & \leq \int_{0}^{T}\left\|(\hat{\pi} \circ \gamma)^{\prime}(t)\right\| \mathrm{d} t \\
& =\int_{0}^{T}\left\|\pi \circ \omega_{\mathbb{H}}\left(\gamma^{\prime}(t)\right)\right\| \mathrm{d} t \\
& \leq \int_{0}^{T}\left\|\omega_{\mathbb{H}}\left(\gamma^{\prime}(t)\right)\right\| \mathrm{d} t \\
& =\ell(\gamma)=d(0, p) .
\end{aligned}
$$

In the first equality, we used the fact that $\hat{\pi}$ is a morphism of Lie groups and its differential is $\pi$, i.e., $\omega_{\mathbb{H} /[\mathbb{H}, \mathbb{H}]} \circ \mathrm{d} \hat{\pi}=\pi \circ \omega_{\mathbb{H}}$, where $\omega_{\mathbb{H} /[\mathbb{H}, \mathbb{H}]}$ is the Mauer-Cartan form of $\mathbb{H} /[\mathbb{H}, \mathbb{H}]$.

Proposition 2.2 Let $d, d^{\prime}$ be two subRiemannian metrics on $\mathbb{H}$ such that

$$
\lim _{p \rightarrow \infty} \frac{d(0, p)}{d^{\prime}(0, p)}=1 .
$$

Then,

$$
\hat{\pi}(\{p \in \mathbb{H}: d(0, p) \leq R\})=\hat{\pi}\left(\left\{p \in \mathbb{H}: d^{\prime}(0, p) \leq R\right\}\right) .
$$

Proof Let $\|\cdot\|$ and $\|\cdot\|^{\prime}$ be the norms on $\mathfrak{h} /[\mathfrak{h}, \mathfrak{h}]$ induced by $d$ and $d^{\prime}$, respectively. We will show that

$$
\lim _{v \rightarrow \infty} \frac{\|v\|}{\|v\|^{\prime}}=1
$$


which easily implies $\|\cdot\|=\|\cdot\|^{\prime}$, because for any fixed $v \in \mathfrak{h} /[\mathfrak{h}, \mathfrak{h}]$ one has $1=$ $\lim _{t \rightarrow \infty} \frac{\|t v\|}{\|t v\|^{\prime}}=\frac{\|v\|}{\|v\|^{\prime}}$. Moreover, the equality (4) follows from Proposition 2.1 combined with (3) and (5).

Since both maps $\hat{\pi}:(\mathbb{H}, d) \rightarrow(\mathfrak{h} /[\mathfrak{h}, \mathfrak{h}],\|\cdot\|)$ and $\hat{\pi}:\left(\mathbb{H}, d^{\prime}\right) \rightarrow\left(\mathfrak{h} /[\mathfrak{h}, \mathfrak{h}],\|\cdot\|^{\prime}\right)$ are submetries, for every $v \in \mathfrak{h} /[\mathfrak{h}, \mathfrak{h}]$ there are $p_{v}, p_{v}^{\prime} \in \mathbb{H}$ such that $\hat{\pi}\left(p_{v}\right)=\hat{\pi}\left(p_{v}^{\prime}\right)=v$, $\|v\|=d\left(0, p_{v}\right)$ and $\|v\|^{\prime}=d^{\prime}\left(0, p_{v}^{\prime}\right)$.

Moreover, it holds $\|v\|^{\prime} \leq d^{\prime}\left(0, p_{v}\right)$ and $\|v\| \leq d\left(0, p_{v}^{\prime}\right)$, again because $\hat{\pi}$ is a submetry in both cases. Therefore,

$$
\frac{d\left(0, p_{v}\right)}{d^{\prime}\left(0, p_{v}\right)} \leq \frac{\|v\|}{\|v\|^{\prime}} \leq \frac{d\left(0, p_{v}^{\prime}\right)}{d^{\prime}\left(0, p_{v}^{\prime}\right)}
$$

Finally, if $v \rightarrow \infty$, then both $d\left(0, p_{v}\right)$ and $d\left(0, p_{v}^{\prime}\right)$ go to infinity as well. The relation (5) is thus proven.

\subsection{Balayage area and lifting of curves}

Let $V \subset \mathfrak{h}$ be a two-dimensional subspace with $V \cap[\mathfrak{h}, \mathfrak{h}]=\{0\}$. Then, $[\mathfrak{h}, \mathfrak{h}]=[V, V]$, i.e., $V$ is bracket generating. Moreover, $\left.\pi\right|_{V}: V \rightarrow \mathfrak{h} /[\mathfrak{h}, \mathfrak{h}]$ is an isomorphism.

If $\rho:[0, T] \rightarrow \mathfrak{h} /[\mathfrak{h}, \mathfrak{h}]$ is a curve with $\rho(0)=0$, then there is a unique $\tilde{\rho}:[0, T] \rightarrow \mathbb{H}$ such that

$$
\left\{\begin{array}{l}
\tilde{\rho}(0)=0, \\
\omega_{\mathbb{H}}\left(\tilde{\rho}^{\prime}(t)\right)=\left.\pi\right|_{V} ^{-1}\left(\rho(t)^{\prime}\right) .
\end{array}\right.
$$

Since $(\pi \circ \tilde{\rho})^{\prime}=\rho^{\prime}$, then $\pi \circ \tilde{\rho}=\rho$. So, $\tilde{\rho}$ is called the lift of $\rho$.

The previous ODE system that defines $\tilde{\rho}$ can be easily integrated. Let $X, Y \in V$ be a basis, set $Z:=[X, Y]$, so that $(X, Y, Z)$ is a basis of $\mathfrak{h}$. Let $(x, y, z)=\exp (x X+y Y+z Z)$ be the exponential coordinates on $\mathbb{H}$ defined by $(X, Y, Z)$. Using the Backer-Campbell-Hausdorff formula, one shows that $X, Y, Z$ induce the following left-invariant vector fields on $\mathbb{H}$ :

$$
\hat{X}=\partial_{x}-\frac{y}{2} \partial_{z}, \quad \hat{Y}=\partial_{y}+\frac{x}{2} \partial_{z}, \quad \hat{Z}=\partial_{z} .
$$

Thanks to these vector fields, we can describe the Maurer-Cartan form as

$$
\omega_{\mathbb{H}}(a \hat{X}+b \hat{Y}+c \hat{Z})=a X+b Y+c Z .
$$

The lift of $\rho$ is hence given by the ODE

$$
\left\{\begin{array}{l}
\tilde{\rho}_{1}^{\prime}=\rho_{1}^{\prime}, \\
\tilde{\rho}_{2}^{\prime}=\rho_{2}^{\prime}, \\
\tilde{\rho}_{3}^{\prime}=\frac{1}{2}\left(\rho_{1} \rho_{2}^{\prime}-\rho_{2} \rho_{1}^{\prime}\right) .
\end{array}\right.
$$

Take the coordinates $(x, y)$ on $\mathfrak{h} /[\mathfrak{h}, \mathfrak{h}]$ given by the basis $(\pi(X), \pi(Y))$ and define the balayage area of a curve $\rho:[0, T] \rightarrow \mathfrak{h} /[\mathfrak{h}, \mathfrak{h}]$ as

$$
\mathscr{A}(\rho)=\frac{1}{2} \int_{\rho}(x \mathrm{~d} y-y \mathrm{~d} x) .
$$

If $\rho(0)=0$, then the balayage area of $\rho$ corresponds to the signed area enclosed between the curve $\rho$ and the line passing through 0 and $\rho(T)$.

It follows that

$$
\tilde{\rho}(t)=\left(\rho_{1}(t), \rho_{2}(t), \mathscr{A}\left(\left.\rho\right|_{0} ^{t}\right)\right)
$$


In an implicit form, we can write

$$
\tilde{\rho}(t)=\exp \left(\left(\left.\pi\right|_{V}\right)^{-1}(\rho(t))+\mathscr{A}\left(\left.\rho\right|_{0} ^{t}\right) Z\right) .
$$

Notice that the lift $\tilde{\rho}$ of a curve $\rho$ depends on the choice of $V$. Moreover, both the area and the balayage area in $\mathfrak{h} /[\mathfrak{h}, \mathfrak{h}]$ depend on the choice of the basis $(X, Y)$. Nevertheless, once a plane $V \subset \mathfrak{h}$ is fixed, the lift $\tilde{\rho}$ does not depend on the choice of the basis $X, Y$.

If $g$ is a scalar product on $V$ and $d$ is the corresponding strictly subRiemannian metric, the balayage area gives a characterization of $d$-length-minimizing curves. Let $\bar{g}$ be the scalar product on $\mathfrak{h} /[\mathfrak{h}, \mathfrak{h}]$ induced by $g$. Then, the $d$-length of a curve $\tilde{\rho}:[0, T] \rightarrow \mathbb{H}$ equals the length of $\rho=\pi \circ \tilde{\rho}$.

Therefore, given $p=(x, y, z) \in \mathbb{H}$, we have

$$
d(0, p)=\inf \{\ell(\rho): \rho:[0,1] \rightarrow \mathfrak{h} /[\mathfrak{h}, \mathfrak{h}], \quad \rho(0)=0, \rho(1)=\hat{\pi}(p), \quad \mathscr{A}(\rho)=z\} .
$$

This express the so-called Dido's problem in the plane, and the solutions are arc of circles. It degenerates into a line if $z=0$. We can summarize the last discussion in the following result.

Lemma 2.3 A curve $\tilde{\rho}:[0,1] \rightarrow \mathbb{H}$ is d-length-minimizing from 0 to $p=(x, y, z)$ if and only if $\rho:=\hat{\pi} \circ \tilde{\rho}$ is an arc of a circle from 0 to $\hat{\pi}(p)$ with $\mathscr{A}(\rho)=z$.

\section{Comparison between strictly subRiemannian metrics}

The present section is devoted to comparing strictly subRiemannian metrics. For such metrics, Proposition 3.1 gives the only non-trivial implication in Theorem 1.2. The general case will follow from Proposition 4.1.

Proposition 3.1 Let $d$ and $d^{\prime}$ be two strictly subRiemannian metrics on $\mathbb{H}$ with horizontal spaces $(V, g)$ and $\left(V^{\prime}, g^{\prime}\right)$, respectively. Suppose that there exists a scalar product $\bar{g}$ on $\mathfrak{h} /[\mathfrak{h}, \mathfrak{h}]$ such that both

$$
\left.\pi\right|_{V}:(V, g) \rightarrow(\mathfrak{h} /[\mathfrak{h}, \mathfrak{h}], \bar{g}) \quad \text { and }\left.\pi\right|_{V^{\prime}}:\left(V^{\prime}, g^{\prime}\right) \rightarrow(\mathfrak{h} /[\mathfrak{h}, \mathfrak{h}], \bar{g})
$$

are submetries.

Then,

$$
\sup _{p \in \mathbb{H}}\left|d(0, p)-d^{\prime}(0, p)\right|<\infty
$$

Moreover, if $d \neq d^{\prime}$, then

$$
\limsup _{p \rightarrow \infty}\left|d(0, p)-d^{\prime}(0, p)\right|>0
$$

In the proof, we will give the exact value of the supremum in (8). Indeed, by (11) and (12), we get $\sup _{p \in \mathbb{H}}\left|d(0, p)-d^{\prime}(0, p)\right|=2|h|$, where $h$ is defined below. For (8), we will first prove that two of such subRiemannian metrics are isometric via a conjugation $x \mapsto g x g^{-1}$ for some $g \in \mathbb{H}$, and then, we apply Lemma 3.2. For (9), we will give a sequence $p_{n} \rightarrow \infty$ and a constant $c>0$ such that $\left|d\left(0, p_{n}\right)-d^{\prime}\left(0, p_{n}\right)\right|>c$ for all $n \in \mathbb{N}$. 


\subsection{Proof of (8)}

Since $\operatorname{dim} V=\operatorname{dim} V^{\prime}=2$, then $\left.\pi\right|_{V}$ and $\left.\pi\right|_{V^{\prime}}$ are isomorphisms. Therefore by the assumption, they are isometries onto $(\mathfrak{h} /[\mathfrak{h}, \mathfrak{h}], \bar{g})$.

Let $X \in V \cap V^{\prime}$ be with $g(X, X)=1$. Then, $g^{\prime}(X, X)=1$ as well.

Let $Y \in V$ be orthogonal to $X$ with $g(Y, Y)=1$. Then, $Z:=[X, Y] \neq 0$ and $(X, Y, Z)$ is a basis of $\mathfrak{h}$.

Let $Y^{\prime}:=\left.\pi\right|_{V^{\prime}} ^{-1}(\pi(Y)) \in V^{\prime}$. Then, $g^{\prime}\left(Y^{\prime}, Y^{\prime}\right)=1$ and $g^{\prime}\left(X, Y^{\prime}\right)=0$. Moreover, there is $h \in \mathbb{R}$ such that $Y^{\prime}=Y+h Z$. In particular, $\left[X, Y^{\prime}\right]=Z$.

Using the formula $\operatorname{Ad}_{\exp (\mathrm{hX})}(\mathrm{v})=\mathrm{e}^{\operatorname{ad}_{\mathrm{hX}}} \mathrm{v}=\mathrm{v}+\mathrm{h}[\mathrm{X}, \mathrm{v}]$, we notice that

$$
\left\{\begin{array}{l}
\operatorname{Ad}_{\exp (\mathrm{hX})}(\mathrm{X})=\mathrm{X}, \\
\operatorname{Ad}_{\exp (\mathrm{hX})}(\mathrm{Y})=\mathrm{Y}^{\prime} \\
\operatorname{Ad}_{\exp (\mathrm{hX})}(\mathrm{Z})=\mathrm{Z} .
\end{array}\right.
$$

In particular, $\left.\operatorname{Ad}_{\exp (\mathrm{hX})}\right|_{\mathrm{V}}:(\mathrm{V}, \mathrm{g}) \rightarrow\left(\mathrm{V}^{\prime}, \mathrm{g}^{\prime}\right)$ is an isometry.

Therefore, the conjugation

$$
C_{\exp (h X)}(p):=\exp (h X) \cdot p \cdot \exp (h X)^{-1}
$$

is an isometry $C_{\exp (h X)}:(\mathbb{H}, d) \rightarrow\left(\mathbb{H}, d^{\prime}\right)$.

We can now use the following Lemma 3.2 and get

$$
\sup _{p \in \mathbb{H}}\left|d(0, p)-d^{\prime}(0, p)\right| \leq 2|h| .
$$

Lemma 3.2 Let $G$ be a group with neutral element e and let $d, d^{\prime}$ be two left-invariant distances on $G$.

If there is $g \in G$ such that for all $p \in G$

$$
d^{\prime}(e, p)=d\left(e, g p g^{-1}\right)
$$

then for all $p \in G$

$$
\left|d(e, p)-d^{\prime}(e, p)\right| \leq 2 \min \left\{d(e, g), d^{\prime}(e, g)\right\} .
$$

Proof Note that since $d$ is left invariant, then for all $a, b \in G$ we have $d(e, a b) \leq d(e, a)+$ $d(e, b)$ and $d(e, a)=d\left(e, a^{-1}\right)$. On the one side, we have $d^{\prime}(e, p)=d\left(e, g p g^{-1}\right) \leq$ $d(e, p)+2 d(e, g)$. On the other side, we have $d(e, p)=d\left(e, g^{-1} g p g^{-1} g\right) \leq d\left(e, g^{-1}\right)+$ $d\left(e, g p g^{-1}\right)+d(e, g)=2 d(e, g)+d^{\prime}(e, p)$. Hence, $\left|d(e, p)-d^{\prime}(e, p)\right| \leq 2 d(e, g)$. Вy symmetry, we have also $\left|d(e, p)-d^{\prime}(e, p)\right| \leq 2 d^{\prime}(e, g)$.

\subsection{Proof of (9)}

We keep the same notation of the previous subsection. Up to switching $V$ with $V^{\prime}$, we can assume $h>0$.

Let $(x, y, z)$ be the exponential coordinates on $\mathbb{H}$ induced by the basis $(X, Y, Z)$ of $\mathfrak{h}$, i.e., $(x, y, z)=\exp (x X+y Y+z Z) \in \mathbb{H}$. Similarly, on $\mathfrak{h} /[\mathfrak{h}, \mathfrak{h}]$ we have coordinates $(x, y)=x \pi(X)+y \pi(Y)$.

For $R>0$, define

$$
p_{R}:=\left(0,2 R, \frac{\pi R^{2}}{2}+2 h R\right)
$$




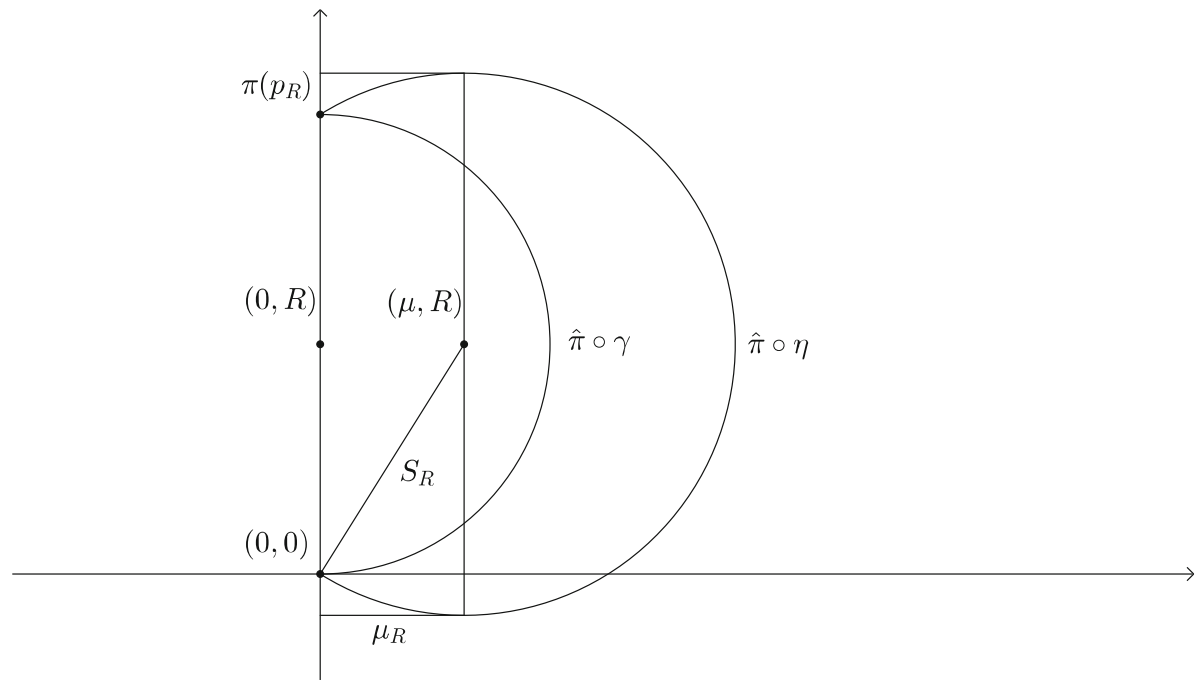

Fig. 1 Curves in $\mathfrak{h} /[\mathfrak{h}, \mathfrak{h}]$ for the proof of (12)

We will show that

$$
\lim _{R \rightarrow \infty} d\left(0, p_{R}\right)-d^{\prime}\left(0, p_{R}\right)=2 h .
$$

Fix $R>0$. Let $\gamma:[0, T] \rightarrow \mathbb{H}$ be a $d^{\prime}$-minimizing curve from 0 to $p_{R}$. Then, $\hat{\pi} \circ \gamma:$ $[0, T] \rightarrow \mathfrak{h} /[\mathfrak{h}, \mathfrak{h}]$ is half circle of center $(0, R)$ and radius $R$, see Fig. 1 . The balayage area of $\hat{\pi} \circ \gamma$ is

$$
\mathscr{A}(\hat{\pi} \circ \gamma)=\frac{\pi R^{2}}{2} .
$$

Let $\eta:[0, T] \rightarrow \mathbb{H}$ be the $d$-length-minimizing curve from 0 to $p_{R}$. Then, $\hat{\pi} \circ \eta$ : $[0, T] \rightarrow \mathfrak{h} /[\mathfrak{h}, \mathfrak{h}]$ is an arc of a circle of radius $S_{R}$ whose balayage area is

$$
\mathscr{A}(\hat{\pi} \circ \eta)=\frac{\pi R^{2}}{2}+2 R h=\mathscr{A}(\hat{\pi} \circ \gamma)+2 R h .
$$

It is clear that $S_{R}>R$ and that the circle of $\hat{\pi} \circ \eta$ has center $\left(\mu_{R}, R\right)$ for some $\mu_{R}>0$. So we have

$$
S_{R}^{2}=R^{2}+\mu_{R}^{2}
$$

It is also clear from the picture that

$$
\frac{\pi S_{R}^{2}}{2}+2 R \mu_{R} \leq \mathscr{A}(\hat{\pi} \circ \eta) \leq \frac{\pi S_{R}^{2}}{2}+2 S_{R} \mu_{R} .
$$

Now, let's look at the lengths. First of all, notice that $\ell_{d^{\prime}}(\gamma)=\ell(\hat{\pi} \circ \gamma)$ and $\ell_{d}(\eta)=$ $\ell(\hat{\pi} \circ \eta)$. For one curve, we have

$$
\ell(\hat{\pi} \circ \gamma)=\pi R
$$

and for the other, we have the estimate

$$
\pi S_{R}+2 \mu_{R} \leq \ell(\hat{\pi} \circ \eta)
$$


which is clear from the picture. Hence,

$$
\begin{aligned}
\liminf _{R \rightarrow \infty} d(0, p)-d^{\prime}(0, p) & =\liminf _{R \rightarrow \infty} \ell(\hat{\pi} \circ \eta)-\ell(\hat{\pi} \circ \gamma) \\
& \geq \lim _{R \rightarrow \infty} \pi S_{R}+2 \mu_{R}-\pi R \\
& =\lim _{R \rightarrow \infty} \pi\left(S_{R}-R\right)+2 \mu_{R} .
\end{aligned}
$$

We claim that

$$
\lim _{R \rightarrow \infty} \pi\left(S_{R}-R\right)+2 \mu_{R}=2 h .
$$

Let us start by checking that,

$$
\mu_{R}<h .
$$

Indeed, from the first inequality of (14) together with (13) it follows

$$
\frac{\pi S_{R}^{2}}{2}+2 R \mu_{R} \leq \frac{\pi R^{2}}{2}+2 h R .
$$

Since $S_{R}>R$, then

$$
0<\frac{\pi S_{R}^{2}}{2}-\frac{\pi R^{2}}{2} \leq 2 R\left(h-\mu_{R}\right),
$$

i.e., the inequality (16).

From the second inequality of (14) together with (13), we get

$$
\frac{\pi R^{2}}{2}+2 R h \leq \frac{\pi S_{R}^{2}}{2}+2 S_{R} \mu_{R}
$$

Using the facts $\mu_{R} \leq h$ and $S_{R} \leq R+\mu_{R} \leq R+h$, from the above inequality one gets

$$
\begin{aligned}
0 \leq 2\left(h-\mu_{R}\right) & \leq\left(S_{R}-R\right) \frac{1}{R}\left(\frac{\pi}{2}\left(S_{R}+R\right)+2 \mu_{R}\right) \\
& \leq\left(S_{R}-R\right)\left(\pi+\frac{h}{R}\left(\frac{\pi}{2}+2\right)\right)
\end{aligned}
$$

Moreover, since $h^{2} \geq \mu_{R}^{2}=S_{R}^{2}-R^{2}=\left(S_{R}-R\right)\left(S_{R}+R\right)$, we also have

$$
\lim _{R \rightarrow \infty}\left(S_{R}-R\right)=0 .
$$

Finally, from (18) and (17) we obtain (15), as claimed. This completes the proof of (12) and of Proposition 3.1.

\section{Comparison between Riemannian and strictly subRiemannian metrics}

Let $d_{R}$ be a Riemannian metric on $\mathbb{H}$ with horizontal space $(\mathfrak{h}, g)$.

Let $V \subset \mathfrak{h}$ be the plane orthogonal to $[\mathfrak{h}, \mathfrak{h}]$ and let $d_{C C}$ be the strictly subRiemannian metric on $\mathbb{H}$ with horizontal space $\left(V,\left.g\right|_{V}\right)$.

Fix a basis $(X, Y, Z)$ for $\mathfrak{h}$ such that $(X, Y)$ is an orthonormal basis of $\left(V,\left.g\right|_{V}\right)$ and $Z=[X, Y]$. The matrix representation of $g$ with respect to $(X, Y, Z)$ is

$$
g=\left(\begin{array}{ccc}
1 & 0 & 0 \\
0 & 1 & 0 \\
0 & 0 & \zeta^{2}
\end{array}\right)
$$


where $\zeta>0$.

Let $d_{C C}$ be the strictly subRiemannian metric on $\mathbb{H}$ with horizontal space $\left(V,\left.g\right|_{V}\right)$.

Our aim in this section is to prove the following proposition.

Proposition 4.1 If $d_{C C}(0, p)$ is large enough, then:

$$
0 \leq d_{C C}(0, p)-d_{R}(0, p) \leq \frac{4 \pi^{2}}{\zeta^{2}} \frac{1}{d_{C C}(0, p)-\frac{2^{3 / 2} \pi}{\zeta}} .
$$

In particular, it holds

$$
\lim _{p \rightarrow \infty}\left|d_{C C}(0, p)-d_{R}(0, p)\right|=0 .
$$

For the proof of this statement, we need to know length-minimizing curves for $d_{R}$ and $d_{C C}$, and a few properties of those, see the exposition in "Appendix."

Proof Let $(x, y, z)$ be the exponential coordinates on $\mathbb{H}$ induced by the basis $(X, Y, Z)$ of $\mathfrak{h}$, i.e., $(x, y, z)=\exp (x X+y Y+z Z) \in \mathbb{H}$. Fix $p=\left(p_{1}, p_{2}, p_{3}\right) \in \mathbb{H}$.

Notice that both $d_{R}$ and $d_{C C}$ are generated as length metrics using the same length measure $\ell$, with the difference that $d_{R}$ minimizes the length among all the curves, while $d_{C C}$ takes into account only the curves tangent to $V$. This implies that

$$
\forall p, q \in \mathbb{H} \quad d_{C C}(p, q) \geq d_{R}(p, q) ;
$$

therefore, we get the first inequality in (19). We need to prove the second inequality of (19).

If $p \in\{z=0\}$, then $d_{C C}(0, p)=d_{R}(0, p)$ by Corollary 5.9, and the claim is true.

Suppose $p \notin\{z=0\}$ and let $\gamma:[0, T] \rightarrow \mathbb{H}$ be a $d_{R}$-length-minimizing curve from $0=\gamma(0)$ to $p=\gamma(T)$. Since $p \notin\{z=0\}$ and since we supposed that $d_{C C}(0, p)$ is large enough, then by Corollary 5.7 we can parametrize $\gamma$ in such a way that $\gamma$ is exactly in the form expressed in TYPE II in Proposition 5.4 for some $k>0$ and $\theta \in \mathbb{R}$.

By Corollary 5.6, it holds

$$
k T \leq 2 \pi
$$

Moreover, by Corollary 5.10

$$
d_{R}(0, p)=\left\|\omega_{\mathbb{H}}\left(\gamma^{\prime}\right)\right\| \cdot T=\sqrt{1+\frac{k^{2}}{\zeta^{2}}} \cdot T .
$$

Let $\eta:[0, T] \rightarrow \mathbb{H}$ be the $d_{C C}$-length-minimizing curve corresponding to $\gamma$ as shown in Corollary 5.10. Then, we know that $d_{C C}(0, \eta(T))=\ell(\eta)=T$, and

$$
p=\gamma(T)=\eta(T)+\left(0,0, \frac{k T}{\zeta^{2}}\right) .
$$

Hence, by Corollary 5.10 and (21)

$$
d_{C C}(0, p) \leq d_{C C}(0, \eta(T))+d_{C C}(\eta(T), \gamma(T)) \leq T+\frac{2^{3 / 2} \pi}{\zeta},
$$

i.e.,

$$
\frac{1}{T} \leq \frac{1}{d_{C C}(0, p)-\frac{2^{3 / 2} \pi}{\zeta}} .
$$

Since $\eta$ is a $d_{C C}$-rectifiable curve, then $\eta(T)_{3}=\mathscr{A}(\hat{\pi} \circ \eta)$, where $\eta(T)_{3}$ is the third coordinate of the point in the exponential coordinates. Since $\hat{\pi} \circ \gamma=\hat{\pi} \circ \eta$, then we have 
by (23)

$$
p_{3}=\mathscr{A}(\hat{\pi} \circ \gamma)+\frac{k T}{\zeta^{2}}
$$

Notice that $\hat{\pi} \circ \gamma$ is an arc of a circle in $\mathfrak{h} /[\mathfrak{h}, \mathfrak{h}]$ of radius $\frac{1}{k}$, see Proposition 5.4.

Now we want to define a horizontal curve $\tilde{\rho}:[-\epsilon, T+\epsilon] \rightarrow \mathbb{H}$, where $\epsilon>0$ has to be chosen, such that $\tilde{\rho}(-\epsilon)=0$ and $\tilde{\rho}(T+\epsilon)=p$. We first define a curve $\rho:[-\epsilon, T+\epsilon] \rightarrow$ $\mathfrak{h} /[\mathfrak{h}, \mathfrak{h}]$ and then take its lift to $\mathbb{H}$.

For the definition of $\rho$, we follow two different strategies for two different cases (see Fig. 2):

Case 1. Suppose that $\hat{\pi} \circ \gamma$ doesn't cover the half of the circle, i.e., $T \leq \frac{\pi}{k}$. Set $\lambda=$ $\hat{\pi}(p) \in \mathfrak{h} /[\mathfrak{h}, \mathfrak{h}]$. Then, $T$ is smaller than the circle of diameter $\|\lambda\|$, i.e.,

$$
\|\lambda\| \geq \frac{T}{\pi} .
$$

Let $\lambda^{\perp} \in \mathfrak{h} /[\mathfrak{h}, \mathfrak{h}]$ be the unit vector perpendicular to $\lambda$ and forming an angle smaller than $\pi / 2$ with the $\operatorname{arc} \hat{\pi} \circ \gamma$.

Let $\epsilon>0$ such that

$$
\epsilon \cdot\|\lambda\|=\frac{k T}{\zeta^{2}}
$$

Now, define $\rho:[-\epsilon, T+\epsilon] \rightarrow \mathfrak{h} /[\mathfrak{h}, \mathfrak{h}]$ as

$$
\rho(t)= \begin{cases}(t+\epsilon) \lambda^{\perp} & \text { for }-\epsilon \leq t \leq 0 \\ \epsilon \lambda^{\perp}+\hat{\pi} \circ \gamma(t) & \text { for } 0 \leq t \leq T \\ \epsilon \lambda^{\perp}+\hat{\pi} \circ \gamma(T)-(t-T) \lambda^{\perp} & \text { for } T \leq t \leq T+\epsilon\end{cases}
$$

Notice that

$$
\mathscr{A}(\rho)=\mathscr{A}(\hat{\pi} \circ \gamma)+\epsilon \cdot\|\lambda\|=\mathscr{A}(\hat{\pi} \circ \gamma)+\frac{k T}{\zeta^{2}} \stackrel{(25)}{=} p_{3}
$$
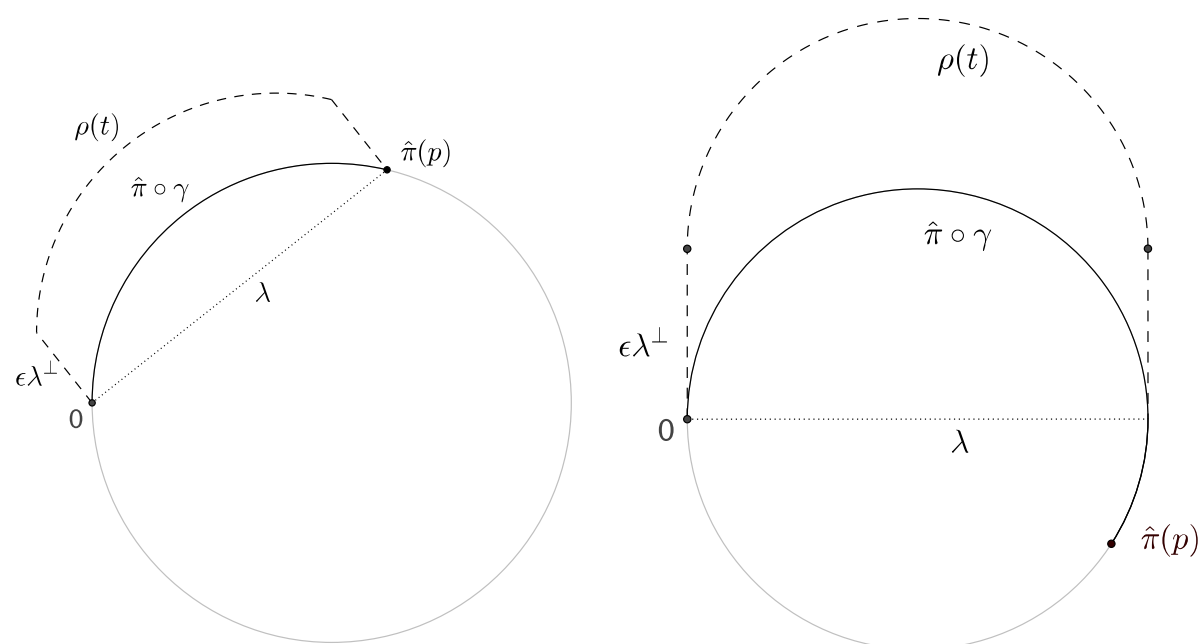

Fig. 2 Curves for case 1 and case 2 in the proof of Proposition 4.1 
and that $\rho(T+\epsilon)=\hat{\pi} \circ \gamma(T)=\hat{\pi}(p)$. Then, the horizontal lift $\tilde{\rho}:[-\epsilon, T+\epsilon] \rightarrow \mathbb{H}$ of $\rho$ is a $d_{C C}$-rectifiable curve from 0 to $p$.

Case 2. Suppose that $\hat{\pi} \circ \gamma$ covers more than half of the circle. Let $\lambda \in \mathfrak{h} /[\mathfrak{h}, \mathfrak{h}]$ be the diameter of the circle that contains 0 . Since $T$ is shorter than the whole circle, then

$$
\|\lambda\| \geq \frac{T}{\pi} .
$$

Let $\lambda^{\perp}$ be the unit vector perpendicular to $\lambda$ and forming an angle smaller than $\pi / 2$ with the $\operatorname{arc} \hat{\pi} \circ \gamma$.

Let $\epsilon>0$ be such that

$$
\epsilon \cdot\|\lambda\|=\frac{k T}{\zeta^{2}}
$$

Now, define $\rho:[-\epsilon, T+\epsilon] \rightarrow \mathfrak{h} /[\mathfrak{h}, \mathfrak{h}]$ as

$$
\rho(t)= \begin{cases}(t+\epsilon) \lambda^{\perp} & \text { for }-\epsilon \leq t \leq 0 \\ \epsilon \lambda^{\perp}+\hat{\pi} \circ \gamma(t) & \text { for } 0 \leq t \leq \frac{\pi\|\lambda\|}{2} \\ \epsilon \lambda^{\perp}+\lambda-\left(t-\frac{\pi\|\lambda\|}{2}\right) \lambda^{\perp} & \text { for } \frac{\pi\|\lambda\|}{2} \leq t \leq \frac{\pi\|\lambda\|}{2}+\epsilon \\ \hat{\pi} \circ \gamma(t-\epsilon) & \text { for } \frac{\pi\|\lambda\|}{2}+\epsilon \leq t \leq T+\epsilon\end{cases}
$$

where we used the fact $\lambda=\hat{\pi} \circ \gamma\left(\frac{\pi\|\lambda\|}{2}\right)$. Notice that

$$
\mathscr{A}(\rho)=\mathscr{A}(\hat{\pi} \circ \gamma)+\epsilon \cdot\|\lambda\|=\mathscr{A}(\hat{\pi} \circ \gamma)+\frac{k T}{\zeta^{2}}=p_{3} .
$$

Then, the horizontal lift $\tilde{\rho}:[-\epsilon, T+\epsilon] \rightarrow \mathbb{H}$ of $\rho$ is a $d_{C C}$-rectifiable curve from 0 to $p$.

In both cases, $\tilde{\rho}$ is a horizontal curve from 0 to $p$ of length

$$
\ell(\tilde{\rho})=T+2 \epsilon
$$

Moreover, from (26) and (27) [respectively (28) and (29)] we get

$$
\epsilon=\frac{k T}{\zeta^{2}\|\lambda\|} \leq \frac{k T}{\zeta^{2}} \frac{\pi}{T} \stackrel{(21)}{\leq} \frac{2 \pi}{\zeta^{2}} \frac{\pi}{T}=\frac{2 \pi^{2}}{\zeta^{2} T}
$$

Finally using in order (22), (30), (31), (24)

$$
\begin{aligned}
& d_{C C}(0, p)-d_{R}(0, p) \leq \ell(\tilde{\rho})-\sqrt{1+\frac{k^{2}}{\zeta^{2}}} \cdot T \\
& \leq T+2 \epsilon-\sqrt{1+\frac{k^{2}}{\zeta^{2}}} \cdot T \leq 2 \epsilon \leq 2 \frac{2 \pi^{2}}{\zeta^{2} T} \leq \frac{4 \pi^{2}}{\zeta^{2}} \frac{1}{d_{C C}(0, p)-\frac{2^{3 / 2} \pi}{\zeta}} .
\end{aligned}
$$

Remark 4.2 The inequality (2) is sharp. Indeed, for $z \rightarrow \infty$, we have the asymptotic equivalence

$$
d_{C C}(0,(0,0, z))-d_{R}(0,(0,0, z)) \sim \frac{4 \pi^{2}}{\zeta^{2}} \frac{1}{d_{C C}(0,(0,0, z))} .
$$

Proof of (32) We claim that, for $z>0$ large enough,

$$
d_{R}(0,(0,0, z))=2 \sqrt{\pi} \sqrt{z-\frac{\pi}{\zeta^{2}}} .
$$


Let $\gamma:[0, T] \rightarrow \mathbb{H}$ be a $d_{R}$-length-minimizing curve from 0 to $(0,0, z)$. Since $z$ is large, we assume that $\gamma$ is of (TYPE II), see Proposition 5.4, for some $k>0$ and $\theta=0$. Since the end point is on the $Z$-axis, we have

$$
k T=2 \pi
$$

and $z=\frac{T}{2 k}+\frac{k T}{\zeta^{2}}$, from which follows

$$
T^{2}=4 \pi\left(z-\frac{2 \pi}{\zeta^{2}}\right) .
$$

We know also the length of $\gamma$ (see Corollary 5.10) and so we get

$$
\begin{aligned}
& d_{R}(0,(0,0, z))=\ell(\gamma)=T\left\|\omega_{\mathbb{H}}\left(\gamma^{\prime}\right)\right\|=T \sqrt{1+\frac{k^{2}}{\zeta^{2}}}=\sqrt{T^{2}+\frac{4 \pi^{2}}{\zeta^{2}}} \\
& =\sqrt{4 \pi\left(z-\frac{2 \pi}{\zeta^{2}}\right)+\frac{4 \pi^{2}}{\zeta^{2}}}=2 \sqrt{\pi} \sqrt{z-\frac{\pi}{\zeta^{2}}} .
\end{aligned}
$$

Claim (33) is proved. From Corollary 5.8, we get $d_{C C}(0,(0,0, z))=2 \sqrt{\pi} \sqrt{z}$ and

$$
\begin{gathered}
d_{C C}(0,(0,0, z))-d_{R}(0,(0,0, z))=2 \sqrt{\pi}\left(\sqrt{z}-\sqrt{z-\frac{\pi}{\zeta^{2}}}\right) \\
=\frac{2 \sqrt{\pi}}{\sqrt{z}} \frac{\frac{\pi}{\zeta^{2}}}{1+\sqrt{1-\frac{\pi}{\zeta^{2} z}}}=\frac{1}{2 \sqrt{\pi} \sqrt{z}} \frac{4 \pi^{2}}{\zeta^{2}} \frac{1}{1+\sqrt{1-\frac{\pi}{\zeta^{2} z}}} .
\end{gathered}
$$

We are now ready to give the proof of the main theorems:

Proof of Theorem 1.2 The implication $(a) \Rightarrow(b)$ is trivial. The implication $(b) \Rightarrow(c)$ is proven in Proposition 2.2. The equivalence $(c) \Leftrightarrow(d)$ follows from Proposition 2.1. The assertion $(e)$ is a restatement of $(d)$. For $(d) \Rightarrow(a)$, one uses Proposition 4.1 in order to reduce to the case when both $d$ and $d^{\prime}$ are strictly subRiemannian, and then, one applies Proposition 3.1.

Proof of Theorem 1.3 This is a consequence of Propositions 4.1 and of the sharpness result (9) of Proposition 3.1.

\section{The horoboundary}

Let $(X, d)$ be a geodesic space and $\mathscr{C}(X)$ the space of continuous functions $X \rightarrow \mathbb{R}$ endowed with the topology of the uniform convergence on compact sets. The map $\iota: X \hookrightarrow \mathscr{C}(X)$, $(\iota(x))(y):=d(x, y)$, is an embedding, i.e., a homeomorphism onto its image.

Let $\mathscr{C}(X) / \mathbb{R}$ be the topological quotient of $\mathscr{C}(X)$ with kernel the constant functions, i.e., for every $f, g \in \mathscr{C}(X)$ we set the equivalence relation $f \sim g \Leftrightarrow f-g$ is constant.

Then, the map $\hat{\imath}: X \hookrightarrow \mathscr{C}(X) / \mathbb{R}$ is still an embedding. Indeed, since the map $\mathscr{C}(X) \rightarrow$ $\mathscr{C}(X) / \mathbb{R}$ is continuous and open, we only need to show that $\hat{\imath}$ is injective: if $x, x^{\prime} \in X$ are such that $\iota(x)-\iota\left(x^{\prime}\right)$ is constant, then one takes $z \in Z$ such that $d(x, z)=d\left(x^{\prime}, z\right)$, which exists because $(X, d)$ is a geodesic space, and checks that

$$
d\left(x, x^{\prime}\right)=\iota(x)\left(x^{\prime}\right)-\iota\left(x^{\prime}\right)\left(x^{\prime}\right)=\iota(x)(z)-\iota\left(x^{\prime}\right)(z)=0 .
$$


Define the horoboundary of $(X, d)$ as

$$
\partial_{h} X:=\operatorname{cl}(\hat{\imath}(X)) \backslash \hat{\imath}(X) \subset \mathscr{C}(X) / \mathbb{R},
$$

where $\operatorname{cl}(\hat{\imath}(X))$ is the topological closure.

Another description of the horoboundary is possible. Fix $o \in X$ and set

$$
\mathscr{C}(X)_{o}:=\{f \in \mathscr{C}(X): f(o)=0\} .
$$

Then, the restriction of the quotient projection $\mathscr{C}(X)_{o} \rightarrow \mathscr{C}(X) / \mathbb{R}$ is an isomorphism of topological vector spaces. Indeed, one easily checks that it is both injective and surjective and that its inverse map is $[f] \mapsto f-f(o)$, where $[f] \in \mathscr{C}(X) / \mathbb{R}$ is the class of equivalence of $f \in \mathscr{C}(X)$.

Hence, we can identify $\partial_{h} X$ with a subset of $\mathscr{C}(X)_{o}$. More explicitly: $f \in \mathscr{C}(X)_{o}$ belongs to $\partial_{h} X$ if and only if there is a sequence $p_{n} \in X$ such that $p_{n} \rightarrow \infty$ (i.e., for every compact $K \subset X$ there is $N \in \mathbb{N}$ such that $p_{n} \notin K$ for all $\left.n>N\right)$ and the sequence of functions $f_{n} \in \mathscr{C}(X)_{o}$,

$$
f_{n}(x):=d\left(p_{n}, x\right)-d\left(p_{n}, o\right),
$$

converge uniformly on compact sets to $f$.

Proof of Corollary 1.4 Let us first remark that if $d, d^{\prime}$ are two geodesic distances on $X$ and

$$
\lim _{d(p, q)+d^{\prime}(p, q) \rightarrow \infty}\left|d^{\prime}(p, q)-d(p, q)\right|=0 .
$$

then

$$
\partial_{h}\left(X, d^{\prime}\right)=\partial_{h}(X, d) .
$$

Indeed, first of all the space $\mathscr{C}(X)_{o}$ depends only on the topology of $X$. Moreover, if $f \in$ $\partial_{h}(X, d)$, let $p_{n} \in X$ be a sequence as in (36) and set $f_{n}^{\prime}(x):=d^{\prime}\left(p_{n}, x\right)-d^{\prime}\left(p_{n}, o\right)$. Then,

$$
\left|f_{n}^{\prime}(x)-f_{n}(x)\right| \leq\left|d^{\prime}\left(p_{n}, x\right)-d\left(p_{n}, x\right)\right|+\left|d^{\prime}\left(p_{n}, o\right)-d\left(p_{n}, o\right)\right|,
$$

and as a consequence of (37) we get $f_{n}^{\prime} \rightarrow f$ uniformly on compact sets. This shows $\partial_{h}(X, d) \subset \partial_{h}\left(X, d^{\prime}\right)$. The other inclusion follows by the symmetry of (37) in $d$ and $d^{\prime}$.

Now, if $d_{R}$ and $d_{C C}$ are metrics on $\mathbb{H}$ like in Corollary 1.4, then (37) is easily satisfied thanks to Theorem 1.3, and therefore, $\partial_{h}\left(\mathbb{H}, d_{R}\right)=\partial_{h}\left(\mathbb{H}, d_{C C}\right)$ if the Riemannian metric $d_{R}$ and the subRiemannian metric $d_{C C}$ are compatible. The conclusion follows from [18].

The Busemann points in the boundary $\partial_{h}(X, d)$ are usually defined as the horofunctions associated with sequences of points $\left(p_{n}\right)$ diverging to infinity along rays or "almost geodesic rays". However, in the literature there are different definitions of almost geodesic rays, according to the generality of the metric space $(X, d)$ under consideration $([9,14,25])$. A map $\gamma: I=[0,+\infty) \rightarrow(X, d)$ into a complete length space is called

- a quasi-ray, if the length excess

$$
\Delta_{N}(\gamma)=\sup _{t, s \in[N,+\infty)} \ell(\gamma ; t, s)-d(\gamma(t), \gamma(s))
$$

tends to zero for $N \rightarrow+\infty$;

- an almost geodesic ray, if

$$
\Theta_{N}(\gamma)=\sup _{t, s \in[N,+\infty)} d(\gamma(t), \gamma(s))+d(\gamma(s), \gamma(0))-t
$$

tends to zero for $N \rightarrow+\infty$. 
(Notice that the second definition depends on the parametrization, while the first one is intrinsic.) We will use here a notion of Busemann points which is more general than both of them:

Definition 5.1 A sequence of points $\left(p_{n}\right)$ diverging to infinity in $(X, d)$ is said to diverge almost straightly if for all $\epsilon>0$, there exists $L$ such that for every $n \geq m \geq L$ we have

$$
d\left(p_{L}, p_{m}\right)+d\left(p_{m}, p_{n}\right)-d\left(p_{L}, p_{n}\right)<\epsilon
$$

It is easy to verify that points diverging along a quasi-ray or along an almost geodesic ray diverge almost straightly. We then define a Busemann point as a horofunction $f$ which is the limit of a sequence $f_{n}(x)=d\left(p_{n}, x\right)-d\left(p_{n}, o\right)$, for points $\left(p_{n}\right)$ diverging to infinity almost straightly.

To prove Corollary 1.5, we need the following lemma. We remind that a metric space is boundedly compact if closed balls are compact.

Lemma 5.2 Let $(X, d)$ be a boundedly compact geodesic space, $o \in X$ and $\left\{p_{n}\right\}_{n \in \mathbb{N}} \subset X a$ sequence of points diverging almost straightly. Then:

(i) the sequence $f_{n}(x)=d\left(p_{n}, x\right)-d\left(p_{n}, o\right)$ converges uniformly on compacts to a horofunction $f$;

(ii) $\lim _{n \rightarrow \infty} f\left(p_{n}\right)+d\left(o, p_{n}\right)=0$.

Proof Since the 1-Lipschitz functions $f_{n}$ are uniformly bounded on compact sets and $(X, d)$ is boundedly compact, then the family $\left\{f_{n}\right\}_{n \in \mathbb{N}}$ is pre-compact with respect to the uniform convergence on compact sets. Hence, if we prove that there is a unique accumulation point, then we obtain that the whole sequence $\left\{f_{n}\right\}_{n \in \mathbb{N}}$ converges.

So, let $g, g^{\prime} \in \mathscr{C}(X)$ and let $\left\{f_{n_{k}}\right\}_{k \in \mathbb{N}}$ and $\left\{f_{n_{k}^{\prime}}\right\}_{k \in \mathbb{N}}$ be two subsequences of $\left\{f_{n}\right\}_{n \in \mathbb{N}}$ such that $f_{n_{k}} \rightarrow g$ and $f_{n_{k}^{\prime}} \rightarrow g^{\prime}$ uniformly on compact sets. We claim

$$
\forall \epsilon>0 \exists R_{\epsilon} \in \mathbb{R} \forall x \in X \quad\left|g^{\prime}(x)+R_{\epsilon}-g(x)\right| \leq \epsilon .
$$

Let $\epsilon>0$. Let $L \in \mathbb{N}$ be such that (38) holds for every $n \geq m \geq L$. Define for $x \in X$

$$
\begin{aligned}
& g_{L}(x):=\lim _{k \rightarrow \infty} d\left(p_{n_{k}}, x\right)-d\left(p_{n_{k}}, p_{L}\right)=g(x)-g\left(p_{L}\right) \\
& g_{L}^{\prime}(x):=\lim _{k \rightarrow \infty} d\left(p_{n_{k}^{\prime}}, x\right)-d\left(p_{n_{k}^{\prime}}, p_{L}\right)=g^{\prime}(x)-g^{\prime}\left(p_{L}\right) .
\end{aligned}
$$

Then, for $n_{i} \geq n_{j}^{\prime} \geq L$, we get for all $x \in X$

$$
\begin{gathered}
d\left(p_{n_{i}}, x\right)-d\left(p_{n_{i}}, p_{L}\right)-d\left(p_{n_{j}^{\prime}}, x\right)+d\left(p_{n_{j}^{\prime}}, p_{L}\right) \\
\quad \leq d\left(p_{n_{i}}, p_{n_{j}^{\prime}}\right)-d\left(p_{n_{i}}, p_{L}\right)+d\left(p_{n_{j}^{\prime}}, p_{L}\right) \leq \epsilon .
\end{gathered}
$$

By taking the limit $i \rightarrow \infty$ and $j \rightarrow \infty$, we obtain for all $x \in X$

$$
g_{L}(x)-g_{L}^{\prime}(x) \leq \epsilon .
$$

By the symmetry of the argument, also $g_{L}^{\prime}(x)-g_{L}(x) \leq \epsilon$ holds. Therefore for all $x \in X$

$$
\epsilon \geq\left|g_{L}^{\prime}(x)-g_{L}(x)\right|=\left|g^{\prime}(x)-g(x)+g\left(p_{L}\right)-g^{\prime}\left(p_{L}\right)\right| .
$$

Setting $R_{\epsilon}=g\left(p_{L}\right)-g^{\prime}\left(p_{L}\right)$, we conclude the proof of claim (39). 
It is now easy to conclude ( $i$ ) from (39). Indeed, taking $x=o$, we have $\left|R_{\epsilon}\right| \leq \epsilon$; therefore, for all $\epsilon>0$ and for all $x \in X\left|g(x)-g^{\prime}(x)\right| \leq 2 \epsilon$, i.e., $g=g^{\prime}$. This completes the proof of $(i)$.

To prove assertion (ii), fix $\epsilon>0$ and let $L \in \mathbb{N}$ be as above. Then, we have for all $n \geq m \geq L$

$$
\begin{aligned}
0 \leq & d\left(p_{m}, p_{n}\right)-d\left(p_{n}, o\right)+d\left(p_{m}, o\right) \\
= & d\left(p_{m}, p_{n}\right)+d\left(p_{L}, p_{m}\right)-d\left(p_{L}, p_{n}\right) \\
& +d\left(p_{L}, p_{n}\right)-d\left(p_{n}, o\right)-d\left(p_{L}, p_{m}\right)+d\left(p_{m}, o\right) \\
\leq & \epsilon+d\left(p_{L}, p_{n}\right)-d\left(p_{n}, o\right)-d\left(p_{L}, p_{m}\right)+d\left(p_{m}, o\right) .
\end{aligned}
$$

Taking first the limit $n \rightarrow \infty$ and then $m \rightarrow \infty$ in the above lines, we obtain the estimate

$$
\begin{aligned}
0 & \leq \liminf _{m \rightarrow \infty} f\left(p_{m}\right)+d\left(p_{m}, o\right) \\
& \leq \limsup _{m \rightarrow \infty} f\left(p_{m}\right)+d\left(p_{m}, o\right) \leq \epsilon+f\left(p_{L}\right)-f\left(p_{L}\right)=\epsilon .
\end{aligned}
$$

Since $\epsilon>0$ is arbitrary, then $(i i)$ holds true.

Then, the proof of Corollary 1.5 runs similarly to Theorem 6.5 of [18].

Proof of Corollary 1.5 The horofunctions of type (nv) clearly are Busemann points, as they are limits, in particular, of the Riemannian geodesic rays which are the horizontal half lines issued from the origin and which are always minimizing, see Proposition 5.4 and Corollary 5.5 in "Appendix." On the other hand, consider a horofunction of type $(\mathbf{v}), h_{u}=(v, z)=$ $|u|-|u-v|$, for $u \in W$. Assume that there exists an almost straightly diverging sequence of points $p_{n}=v_{n}+z_{n} Z$ converging to $h_{u}$. By Lemma 5.2 (ii), we deduce that

$$
\lim _{n \rightarrow \infty} f_{u}\left(p_{n}\right)+d_{R}\left(o, p_{n}\right)=\lim _{n \rightarrow \infty}|u|-\left|u-v_{n}\right|+d_{R}\left(o, p_{n}\right)=0 ;
$$

hence, $\left\{v_{n}\right\}_{n \in \mathbb{N}}$ is necessarily an unbounded sequence. By Corollary 1.4 and the following description of horofunctions, it follows that $h_{u}$ should be of type (nv), a contradiction.

\subsection{Concluding remarks}

The Riemannian Heisenberg group shows a number of counterintuitive features which is worth to stress:

(i) in view of Corollary 1.4, all Riemannian metrics on $\mathbb{H}$ with the same associated asymptotic metric have the same Busemann functions, though they are not necessarily isometric (in contrast, notice that all strictly subRiemannian metrics on $\mathbb{H}$ are isometric). However, this is not surprising, because all left-invariant Riemannian metrics on $\mathbb{H}$ are homothetic.

(ii) there exist diverging sequences of points $\left\{p_{n}\right\}_{n \in \mathbb{N}}$ that visually converge to a limit direction $v$ (that is, the minimizing geodesics $\gamma_{n}$ from $o$ to $p_{n}$ tend to a limit, minimizing geodesic $\gamma_{v}$ with initial direction $v$ ), but whose associated limit point $h_{\left\{p_{n}\right\}}$ is not given by the limit point $\gamma_{v}(+\infty)$ of $\gamma_{v}$. This happens for all vertically divergent sequences $\left\{p_{n}\right\}_{n \in \mathbb{N}}$, as the limit geodesic $\gamma_{v}$ is horizontal in this case (see Proposition 5.4 and Corollary 5.6 in the "Appendix").

(iii) there exist diverging trajectories $\left\{p_{n}\right\}_{n \in \mathbb{N}},\left\{q_{n}\right\}_{n \in \mathbb{N}}$ staying at bounded distance from each other, but defining different limit points (e.g., vertically diverging sequences of points with different limit horofunctions). 
(iv) it is not true that, for a cocompact group of isometries $G$ of $\left(\mathbb{H}, d_{R}\right)$, the limit set of $G$ (which is the set of accumulation points of an orbit $G x_{0}$ in $\partial\left(\mathbb{H}, d_{R}\right)$ ) equals the whole Gromov boundary; for instance, the discrete Heisenberg group $G=\mathbb{H}(\mathbb{Z})$ has a limit set equal to the set of all Busemann points, plus a discrete subset of the interior of the disk boundary $\bar{D}^{2}$. Also, the limit set may depend on the choice of the base point $x_{0} \in \mathbb{H}$.

(v) The functions appearing in (nv) coincide with the Busemann functions of a Euclidean plane in the direction $R_{\vartheta}\left(\hat{v}_{\infty}\right)$; that is, the horofunction $h(v, z)$ associated with a diverging sequence $P_{n}=\left(v_{n}, z_{n}\right)$ of $\left(\mathbb{H}, d_{R}\right)$ is obtained just by dropping the vertical component $z$ of the argument and then applying to $v$ the usual Euclidean Busemann function in the direction which is opposite to the limit direction of the $v_{n}$ 's, rotated by an angle $\vartheta$ depending on the quadratic rate of divergence of the sequence ( $\vartheta$ is zero for points diverging subquadratically, and $\vartheta= \pm \pi$ when the divergence is subquadratical).

These properties mark a remarkable difference to the theory of non-positively curved, simply connected spaces.

Acknowledgements The initial discussions for this work were done at the '2013 Workshop on Analytic and Geometric Group Theory ' in Ventotene. We express our gratitude to the organizers: A. Iozzi, G. Kuhn, and M. Sageev. We also thank the anonymous referee for several improving suggestions.

\section{Appendix: Length-minimizing curves for $d_{C C}$ and $d_{R}$}

In the Heisenberg group, locally length-minimizing curves are smooth solutions of an Hamiltonian system both in the Riemannian and in the subRiemannian case. Locally, lengthminimizing curves are also called geodesics.

Let $d_{R}$ be a Riemannian metric on $\mathbb{H}$ with horizontal space $(\mathfrak{h}, g)$. Let $V \subset \mathfrak{h}$ be the plane orthogonal to $[\mathfrak{h}, \mathfrak{h}]$ and let $d_{C C}$ be the strictly subRiemannian metric on $\mathbb{H}$ with horizontal space $\left(V,\left.g\right|_{V}\right)$.

Fix a basis $(X, Y, Z)$ for $\mathfrak{h}$ such that $(X, Y)$ is an orthonormal basis of $\left(V,\left.g\right|_{V}\right)$ and $Z=[X, Y]$. Set $\zeta=\sqrt{g(Z, Z)}$.

The basis $(X, Y, Z)$ induces the exponential coordinates $(x, y, z)$ on $\mathbb{H}$, i.e., $(x, y, z)=$ $\exp (x X+y Y+z Z)$. We will work in this coordinate system.

The Riemannian and subRiemannian length-minimizing curves are known, and we recall their parametrization in the following two propositions. SubRiemannian geodesics can be found with different notation in [7]. Riemannian geodesics are found in [20], in different coordinates and with parametrization by arc length.

Proposition 5.3 (subRiemannian geodesics) All the non-constant locally length-minimizing curves of $d_{C C}$ starting from 0 and parametrized by arc length are the following: given $k \in \mathbb{R} \backslash\{0\}$ and $\theta \in \mathbb{R}$

(TYPE I) The horizontal lines $t \mapsto(t \cos \theta, t \sin \theta, 0)$;

(TYPE II) The curves $t \mapsto(x(t), y(t), z(t))$ given by

$$
\left\{\begin{array}{l}
x(t)=\frac{1}{k}(\cos \theta(\cos (k t)-1)-\sin \theta \sin (k t)) \\
y(t)=\frac{1}{k}(\sin \theta(\cos (k t)-1)+\cos \theta \sin (k t)) \\
z(t)=\frac{1}{2 k} t-\frac{1}{2 k^{2}} \sin (k t) .
\end{array}\right.
$$


Here the derivative at $t=0$ is $(-\sin \theta, \cos \theta, 0)$.

Proposition 5.4 (Riemannian geodesics) All non-constant locally length-minimizing curves of $d_{R}$ parametrized by a multiple of arc length and starting from 0 are the following: given $k \in \mathbb{R} \backslash\{0\}$ and $\theta \in \mathbb{R}$

(TYPE 0$)$ The vertical line $t \mapsto(0,0, t)$;

(TYPE I) The horizontal lines $t \mapsto(t \cos \theta, t \sin \theta, 0)$;

(TYPE II) The curves $t \mapsto(x(t), y(t), z(t))$ given by

$$
\left\{\begin{array}{l}
x(t)=\frac{1}{k}(\cos \theta(\cos (k t)-1)-\sin \theta \sin (k t)) \\
y(t)=\frac{1}{k}(\sin \theta(\cos (k t)-1)+\cos \theta \sin (k t)) \\
z(t)=\frac{1}{2 k} t-\frac{1}{2 k^{2}} \sin (k t)+\frac{k}{\zeta^{2}} t .
\end{array}\right.
$$

Here the derivative at $t=0$ is $\left(-\sin \theta, \cos \theta, \frac{k}{\zeta^{2}}\right)$, which has Riemannian length $\sqrt{1+\frac{k^{2}}{\zeta^{2}}}$.

The expression of geodesics helps us to prove the following facts.

Corollary 5.5 The horizontal lines of TYPE I are globally $d_{R}$-and $d_{C C}$-length-minimizing curves.

Corollary 5.6 Both $d_{R}$ - and locally $d_{C C}$-length-minimizing curves $\gamma$ of TYPE II are not minimizing from 0 to $\gamma(t)$ if $|t|>\frac{2 \pi}{k}$.

Proof This statement depends on the fact that in both cases, if we fix $k \in \mathbb{R} \backslash\{0\}$, then for all $\theta$ the corresponding length-minimizing curves $\gamma_{k, \theta}$ of TYPE II meet each other at the point $\gamma_{k, \theta}(2 \pi / k)$.

Corollary 5.7 The locally $d_{R}$-length-minimizing curve $\gamma$ of TYPE $0, t \mapsto(0,0, t)$, is not minimizing from 0 to $\gamma(t)$ for $|t|>\frac{2 \pi}{\zeta^{2}}$.

Proof For $k>0$, let $\gamma_{k}$ be the $d_{R}$-length-minimizing curve of TYPE II with this $k$ and $\theta=0$. Then, $\left(\gamma_{k}\right)_{3}\left(\frac{2 \pi}{k}\right)=\frac{\pi}{k^{2}}+\frac{2 \pi}{\zeta^{2}}$. Letting $k \rightarrow \infty$, we obtain $\hat{z}:=\frac{2 \pi}{\zeta^{2}}$. This means that for every $\epsilon>0$ there is $z \leq \hat{z}+\epsilon$ and $k>0$ such that $\gamma_{k}\left(\frac{2 \pi}{k}\right)=(0,0, z)$. Therefore, $t \mapsto(0,0, t)$ cannot be minimizing after $z$ and therefore after $\hat{z}$.

Corollary 5.8 If $p=\left(x, y, p_{3}\right)$ and $q=\left(x, y, q_{3}\right)$, then

$$
d_{C C}(p, q)=2 \sqrt{\pi} \cdot \sqrt{\left|p_{3}-q_{3}\right|} .
$$

Proof First suppose $p=0$ : we have to prove that $d_{C C}(0,(0,0, z))=2 \sqrt{\pi} \sqrt{|z|}$. This is done by looking at the length-minimizing curves: they come from complete circle of perimeter $2 \pi R=d$ and area $\pi R^{2}=|z|$, so that $d_{C C}(0,(0,0, z))=2 \pi \sqrt{\frac{|z|}{\pi}}=2 \sqrt{\pi} \sqrt{|z|}$.

The general case follows from the left invariance of $d_{C C}$ :

$$
\begin{aligned}
d_{C C}\left(\left(x, y, p_{3}\right),\left(x, y, q_{3}\right)\right) & =d_{C C}\left(0,\left(x, y, p_{3}\right)^{-1}\left(x, y, q_{3}\right)\right) \\
& =d_{C C}\left(0,\left(0,0, q_{3}-p_{3}\right)\right) .
\end{aligned}
$$


Corollary 5.9 If $p \in\{z=0\}$, then

$$
d_{C C}(0, p)=d_{R}(0, p)
$$

Corollary $5.10 d_{R}$ - and $d_{C C}$-length-minimizing curves of TYPE II are in bijection via the following rule: If $\eta:[0, T] \rightarrow \mathbb{H}$ is a $d_{C C}$-length-minimizing curve of TYPE II, then

$$
\gamma(t)=\eta(t)+\left(0,0, \frac{k t}{\zeta^{2}}\right)
$$

is $d_{R}$-length-minimizing of TYPE II, where $k \in \mathbb{R}$ is given by $\eta$. Moreover, it holds

$$
\left\|\omega_{\mathbb{H}}\left(\gamma^{\prime}\right)\right\|^{2}=1+\frac{k^{2}}{\zeta^{4}}
$$

and

$$
d_{C C}(\gamma(t), \eta(t))=2 \sqrt{\pi} \sqrt{\frac{k t}{\zeta^{2}}}
$$

Proof All the statements come directly from the expression of the geodesics. Notice that a $d_{C C}$-length-minimizing curve $\eta$ of TYPE II is parametrized by arc length, i.e., $\left\|\omega_{\mathbb{H}}\left(\eta^{\prime}\right)\right\| \equiv 1$.

On the other hand, the corresponding $d_{R}$-length-minimizing curve $\gamma$ has derivative $\omega_{\mathbb{H}}\left(\gamma^{\prime}\right)=\omega_{\mathbb{H}}\left(\eta^{\prime}\right)+\frac{k}{\zeta^{2}} Z$, where $\omega_{\mathbb{H}}\left(\eta^{\prime}\right)$ is orthogonal to $Z$. Hence, $\left\|\omega_{\mathbb{H}}\left(\gamma^{\prime}\right)\right\|^{2}=1+\frac{k^{2}}{\zeta^{4}} \square$

\section{References}

1. Agrachëv, A.A., Barilari, D., Boscain, U.: Introduction to Riemannian and sub-Riemannian geometry (Lecture Notes) (2015). https://webusers.imj-prg.fr/ davide.barilari/Notes.php

2. Ballmann, W.: Lectures on spaces of nonpositive curvature. In: Brin, M. (ed.) DMV Seminar with an Appendix, vol. 25, pp. viii+112, Birkhäuser Verlag, Basel (1995). ISBN: 3-7643- 5242-6

3. Bridson, M.R., Haefliger, A.: Metric spaces of non-positive curvature. In: Grundlehren der Mathematischen Wissenschaften [Fundamental Principles of Mathematical Sciences], vol. 319, pp. xxii+643, Springer, Berlin (1999). ISBN: 3-540-64324-9

4. Breuillard, E., Le Donne, E.: On the rate of convergence to the asymptotic cone for nilpotent groups and subFinsler geometry. Proc. Natl. Acad. Sci. USA 110(48), 19220-19226 (2013). ISSN:1091-6490

5. Burago, D.: Periodic metrics. In: Seminar on Dynamical Systems (St. Petersburg, 1991), vol. 12, pp. 90-95. Progr. Nonlinear Differential Equations Appl. Birkhäuser, Basel (1994)

6. Busemann, H.: The Geometry of Geodesics, p. $x+422$. Academic Press Inc., NewYork, NY (1955)

7. Capogna, L. et al:: An introduction to the Heisenberg group and the sub-Riemannian isoperimetric problem. In: Progress in Mathematics, vol. 259, pp. xvi+223. Birkhäuser Verlag, Basel (2007). ISBN:9783-7643-8132-5; 3-7643-8132-9

8. Cerocchi, F., Sambusetti, A.: Quantitative bounded distance Theorem and Margulis' Lemma for $Z^{n}$ actions with applications to homology. Groups Geom. Dyn. (to appear)

9. Dal'bo, F., Peigné, M., Sambusetti, A.: On the horoboundary and the geometry of rays of negatively curved manifolds. Pac. J. Math. 259(1), 55-100 (2012). ISSN:0030-8730

10. Duchin, M., Shapiro, M.: Rational growth in the Heisenberg group. In: ArXiv e-prints (2014). arXiv: 1411.4201

11. Gromov, M.: Hyperbolic manifolds, groups and actions. In: Riemann Surfaces and Related topics: Proceedings of the 1978 Stony Brook Conference (State Univ. New York, Stony Brook, N.Y., 1978). Annals of Mathematical Studies, vol. 97, pp. 183-213, Princeton Univ. Press, Princeton, NJ (1981)

12. Gromov, M.: Groups of polynomial growth and expanding maps. Inst. Hautes Études Sci. Publ. Math. 53, 53-73 (1981). ISSN:0073-8301

13. Gromov, M.: Hyperbolic groups. In: Essays in Group Theory, vol. 8, pp. 75-263. Math. Sci. Res. Inst. Publ. Springer, New York (1987)

14. Haas, A.: Dirichlet points, Garnett points, and infinite ends of hyperbolic surfaces I. Ann. Acad. Sci. Fenn. Math. 21(1), 3-29 (1996). ISSN:0066-1953 
15. Hadamard, J.: Les surfaces á courbures opposées et leurs lignes géodésiques. Jorn. de Math. 4(5), 27-74 (1898)

16. Jean, F.: Control of nonholonomic systems: from sub-Riemannian geometry to motion planning. In: Springer Briefs in Mathematics, pp. $x+104$. Springer, Cham (2014). ISBN:978- 3-319-08689-7; 978-3319-08690-3

17. Klein, T., Nicas, A.: The horofunction boundary of the Heisenberg group. Pac. J. Math. 242(2), 299-310 (2009). ISSN:0030-8730

18. Klein, T., Nicas, A.: The horofunction boundary of the Heisenberg group: the Carnot-Carathéodory metric. Conform. Geom. Dyn. 14, 269-295 (2010). ISSN:1088-4173

19. Krat, S.A.: Asymptotic properties of the Heisenberg group. In: Zap. Nauchn. Sem. S.-Peterburg. Otdel. Mat. Inst. Steklov. (POMI) 261.Geom. i Topol. 4, vol. 268, pp. 125-154 (2014). ISSN:0373-2703x

20. Marenich, V.: Geodesics in Heisenberg groups. Geom. Dedic. 66(2), 175-185 (1997). ISSN:0046-5755

21. Milnor, J.: A note on curvature and fundamental group. J. Differ. Geom. 2, 1-7 (1968). ISSN:0022-040X

22. Mostow, G.D.: Strong rigidity of locally symmetric spaces. Annals of Mathematics Studies, vol. 78. pp. v+195. Princeton University Press, Princeton, N.J.; University of Tokyo Press, Tokyo (1973)

23. Pansu, P.: Croissance des boules et des géodésiques fermées dans les nilvariétés. Ergod. Theory Dyn. Syst. 3(3), 415-445 (1983). ISSN:0143-3857

24. Patterson, S.J.: The limit set of a Fuchsian group. Acta Math. 136(3-4), 241-273 (1976). ISSN:0001-5962

25. Rieffel, M.A.: Group $C^{*}$-algebras as compact quantum metric spaces. Doc. Math. (electronic) 7, 605-651 (2002). ISSN:1431-0635

26. Rifford, L.: Sub-Riemannian geometry and optimal transport. Springer Briefs in Mathematics, pp. viii+140. Springer, Cham (2014). ISBN:978-3-319-04803-1; 978-3-319-04804-8

27. Roblin, T.: Ergodicité et équidistribution en courbure négative . In: Mém. Soc. Math. Fr. (N.S.), vol. 95, pp. vi+96 (2003). ISSN:0249-633X

28. Stoll, M.: On the asymptotics of the growth of 2-step nilpotent groups. J. Lond. Math. Soc. (2) 58(1), 38-48 (1998). ISSN:0024-6107

29. Sullivan, D.: The density at infinity of a discrete group of hyperbolic motions. Inst. Hautes Études Sci. Publ. Math. 50, 171-202 (1979). ISSN:0073-8301

30. Tits, J.: Free subgroups in linear groups. J. Algebra 20, 250-270 (1972). ISSN:0021-8693

31. Walsh, C.: The horofunction boundary of finite-dimensional normed spaces. Math. Proc. Camb. Philos. Soc. 142(3), 497-507 (2007). ISSN:0305-0041

32. Walsh, C.: The horofunction boundary of the Hilbert geometry. Adv. Geom. 8(4), 503-529 (2008). ISSN:1615-715X

33. Wolf, J.A.: Growth of finitely generated solvable groups and curvature of Riemannian manifolds. J. Differ. Geom. 2, 421-446 (1968). ISSN:0022-040X

34. Webster, C., Winchester, A.: Busemann points of infinite graphs. Trans. Am. Math. Soc. (electronic) 358(9), 4209-4224 (2006). ISSN:0002-9947 